\title{
Matrimonio civil y Constitución Política: la sentencia del Tribunal Constitucional sobre matrimonio para parejas del mismo sexo
}

Este artículo se encuentra disponible para su descarga gratuita en www.anuariocdh.uchile.cl

\section{Jorge Contesse Singh}

Abogado de la Universidad Diego Portales y Máster en Derecho, Yale Law School. Profesor de derecho constitucional, Universidad Diego Portales.

jorge.contesse@udp.cl

\section{RESUMEN}

El trabajo analiza de manera breve la sentencia del Tribunal Constitucional sobre la definición legal de matrimonio en Chile, que lo reserva para un hombre y una mujer. El fallo se pone en el contexto de argumentos que arrancan desde una concepción robusta del derecho a la igual dignidad y revisa los votos de mayoría, minoría y los votos concurrentes, poniendo de relieve algunos problemas de técnica jurídica, como es la prevalencia de votos separados de quienes suscriben la posición mayoritaria en el Tribunal, así como la referencia extensa a fuentes del derecho internacional de los derechos humanos. Se argumenta que, dado el impulso que esa rama del derecho ha experimentado, será cuestión de tiempo para que el Estado deba dar una respuesta constitucional a la demanda que las personas de orientación sexual diversa formulan en clave de derechos fundamentales. No obstante, se afirma, es deber del legislador regular esta cuestión, tal como lo señala la sentencia del Tribunal Constitucional.

Palabras clave: Matrimonio para parejas del mismo sexo-Dignidad - Igualdad - Tribunal Constitucional - Derechos Humanos - Chile

\section{SUMMARY}

This article briefly discusses the Chilean Constitutional Court's decision regarding the definition of marriage in Chile, which reserves the right to marry exclusively to a man and a woman. The article grounds the discussion on a robust conception of the equal dignity of individuals and analyzes the separate opinions of the justices of the Court, emphasizing some technical legal issues such as the prevalence of separate opinions and the references to international human rights law in almost all of them. The article argues that, considering the development of international human rights law and the crafting of claims as fundamental rights claims, it is only a matter of time before the legislature addresses the issue of same-sex marriage, following the Court's statement that it is the legislature's duty to do so.

Key words: Same-sex marriage - Dignity - Equality - Constitutional Court - Human Rights - Chile

"Will you say that the singer can't blow you away? That we hate people just 'cause they're gay"

- "Servitude", Fishbone

\section{Introducción}

En noviembre de 2011, el Tribunal Constitucional de la República de Chile (en adelante "el Tribunal Constitucional", "el Tribunal" o "el TC") dictó sentencia sobre un requerimiento acerca de la aplicabilidad del artículo 102 del Código Civil -la norma que define el contrato de matrimonio-, en 
casos de parejas del mismo sexo. Si bien el Tribunal rechazó el requerimiento, por estimar que excedía su esfera de competencias pronunciarse acerca de una institución completa y compleja como ésta, la mayor parte de sus miembros emitió votos separados, avanzando ideas de fondo sobre la regulación civil del matrimonio en frente de los principios y valores constitucionales. Junto con ello, hubo un voto disidente, que estuvo por acoger el requerimiento, entendiendo que la Constitución ordena extender la posibilidad de celebrar este contrato a parejas no heterosexuales.

En este breve trabajo, exploro las diversas aproximaciones que los ministros del Tribunal adoptaron para abordar esta relevante cuestión. En la demanda por matrimonio para parejas del mismo sexo hay un componente igualitario fuerte que, como ha ocurrido con otras instituciones en épocas pasadas, se enfrenta a concepciones sobre la familia, el matrimonio y, en definitiva, sobre los derechos fundamentales, que requieren ser examinadas. $Y$ es que la sentencia del Tribunal, sin decidir sobre el fondo, emite un juicio importante: es materia del legislador regular el alcance de esta institución, por lo cual están abiertas las posibilidades normativas para enmendar el entendimiento que hasta ahora acompaña a la legislación civil. Si, además, se tiene presente el desarrollo del derecho comparado y del derecho internacional de los derechos humanos, no es aventurado afirmar que la extensión del matrimonio a parejas no heterosexuales es cuestión de tiempo.

En primer lugar, intento circunscribir el asunto que debe considerar el Tribunal, esto es, el examen de la definición de matrimonio civil frente a los estándares de la Constitución, todo ello en el marco de definiciones que mutan sobre la igualdad como derecho fundamental (1). En seguida, examino el caso que decidió el TC, destacando el voto de mayoría, el voto disidente y los votos concurrentes. Sobre estos últimos, observo la prevalencia de citas al derecho internacional de los derechos humanos, no obstante las conclusiones diversas a las que llegan los ministros (2). Concluyo situando el debate local en el contexto de discusiones constitucionales intensas en el derecho comparado, y sugiriendo que una comprensión robusta de la igualdad como derecho fundamental debiera conducir a una regulación igualitaria del matrimonio como institución civil.

\section{El asunto a considerar}

La manera como está regulada la institución civil del matrimonio en el derecho chileno data de mediados del siglo XIX. Fue entonces que, impulsado por el trabajo codificador de Andrés Bello, el Estado chileno normó, primero en el Código Civil de 1857, y luego en la Ley de Matrimonio Civil de 1884, esta institución, determinando su alcance, características y efectos. Es interesante destacar que en el Código de Bello se cristalizó la importancia de la religión para regular el matrimonio, un elemento que, según veremos a propósito de la sentencia del Tribunal Constitucional de noviembre de 2011, mantiene una vigencia desconcertante, habiendo transcurrido más de un siglo desde su adopción ${ }^{1}$. Con la Ley de Matrimonio Civil, por su parte, se avanzó en la regulación de matrimonios no religiosos, legislación que rigió hasta 2004, cuando entró en vigencia un nuevo estatuto sobre la materia ${ }^{2}$.

En lo que interesa a este trabajo, la definición adoptada por el legislador chileno para el matrimonio es categórica: se trata de un contrato que se celebra entre "un hombre y una mujer", con

1 El mensaje con que Andrés Bello sometió a consideración del Congreso Nacional el proyecto de Código Civil es claro en señalar que "[s]e conserva a la autoridad eclesiástica el derecho de decisión sobre la validez del matrimonio y se reconocen como impedimentos para contraerlo los que han sido declarados tales por la Iglesia Católica. El matrimonio que es válido a los ojos de la Iglesia, lo es también ante la ley civil [...]". BELLO, Andrés. "Mensaje del Ejecutivo al Congreso proponiendo la aprobación del Código Civil". Santiago, 22 de noviembre de 1855.

2 Ley No. 19.947 que Establece la Nueva Ley de Matrimonio Civil. Ministerio de Justicia, República de Chile, 17 de mayo de 2004. 
el propósito de "vivir juntos, de procrear, y de auxiliarse mutuamente". Tres constituciones -de 1833, 1925 y 1980 - han regido la vida pública del país, con esta definición de matrimonio en la ley civil. Y si bien los tres textos constitucionales han contenido cláusulas similares reconociendo la igualdad ante la ley de las personas, ha sido solo bajo la vigencia de la actual Carta que se ha impugnado, desde el punto de vista jurídico, la norma civil que define al matrimonio. Lo anterior es bastante obvio, si se atiende al hecho indesmentible que el desafío levantado contra la definición de matrimonio proviene del avance que organizaciones de la sociedad civil y otros grupos han articulado durante las décadas recientes, estimuladas por el desarrollo del derecho internacional de los derechos humanos, entre otros sucesos relevantes ${ }^{3}$.

Lo que me interesa remarcar es que jurídicamente la situación es esencialmente la misma: por un lado, un texto constitucional que entiende a todas las personas dotadas de derechos inalienables, entre los que se cuenta el derecho a ser tratados con igual consideración y, por el otro, una definición legal sobre matrimonio que reserva la institución a contratantes de diferente sexo. Dado que, desde la perspectiva del derecho, el paisaje es el mismo, para comprender la situación que hoy enfrenta Chile (y muchos otros Estados) sobre una regulación desigual del matrimonio, es necesario atender a circunstancias fácticas, como son, por ejemplo, los cambios sociales que experimentan las comunidades políticas y que hacen que los juicios normativos también muten. En tanto la democracia constitucional no entiende otra forma de dar sanción jurídica a esos cambios sociales sino por medio de la ley - "manifestación de la voluntad soberana", según la fórmula de Andrés Bello-, entonces hay un exhorto que hacer al legislador democrático para que efectivamente aborde esta cuestión. No hacerlo es una omisión que cruza la moral, la política y el derecho y que insulta la igual dignidad de las personas, así como el derecho que tenemos de autogobernarnos.

Ahora bien, es evidente que las circunstancias fácticas que varían en el entendimiento que una comunidad política tiene de categorías jurídicas, como es, v.gr., la igualdad ante la ley, son afectadas por "nuevas" valoraciones que se hacen del mundo en que vivimos, así como esas nuevas circunstancias fácticas también influyen en los juicios que realizamos. Y, de este modo, lo que en algún momento se entendía que no estaba en tensión con dicho principio constitucional, más tarde perfectamente puede estarlo. Antes de la impugnación al matrimonio, el campo del derecho de familia ya proveía algunos ejemplos, como lo demuestra la existencia, hasta fines del siglo XX, de categorías de hijos "legítimos", "naturales" e "ilegítimos", a quienes se les reconocían derechos de manera diferenciada, $\mathrm{O}$, hasta nuestros días, la vigencia del régimen de sociedad conyugal, que no entiende a la mujer casada como plenamente capaz para administrar sus bienes propios. Hoy, la impugnación que se hace del matrimonio como un contrato que solo pueden celebrar personas de diferente sexo se suma a la lista de instituciones del derecho de familia que han existido desde que Chile tiene un código sobre la materia y que, siempre, han encontrado tenaz resistencia en diversos grupos que rechazan el progreso y avance de las concepciones de igualdad que el derecho por definición estimula.

¿En qué consisten ellas? ¿Cuáles son las concepciones que hasta hace poco parecían no caber en la comunidad legal y que hoy reclaman un espacio importante? Roberto Gargarella ofrece una mirada que arranca desde el derecho a ser tratados con igual consideración que aplica plenamente a este caso. Según él:

3 "El movimiento gay dio a público [sic] -en nuevos términos y nuevas circunstancias- la vieja cuestión de la justicia. Junto con muchos otros grupos sociales, también los gays pasaron a reivindicar, como derecho, el respeto a su identidad, su libertad y tratamiento no discriminatorio. Esta lucha tuvo una historia peculiar, como cualquier movimiento, pero se inserta en un gran proceso que puede ser identificado como de expansión de la democracia y afirmación de derechos universales". Ver DE LIMA LOPES, José Reinaldo. "El derecho al reconocimiento para gays y lesbianas". Sur-Revista Internacional de Derechos Humanos Año 2 No. 2, 2005, p. 73. 
cualquier intento que haga el poder público de tratar de modo diferente a los iguales, a personas dotadas, como cualquiera de nosotros, de una igual dignidad, requiere de parte del poder coercitivo de un esfuerzo justificativo enorme. Desde el Estado se nos tiene que decir cuál es el argumento extraordinario con que cuentan, para justificar el trato diferente que quieren justificar. No solamente necesitamos que del otro lado haya algún argumento. Si de lo que se trata es de negarle a alguien derechos que le aseguramos a todos los demás, lo que necesitamos de parte del Estado que niega derechos es un esfuerzo justificativo extraordinario, de una razón extraordinaria, para no darle a alguien lo que le damos a todos los demás ${ }^{4}$.

El punto de partida es, así, el derecho a la igualdad, reconocido por las constituciones y por tratados internacionales sobre derechos humanos, en base al cual se articula el reclamo (jurídico) sobre la improcedencia de reservar el matrimonio únicamente a contratantes de sexo diferenciado. Y dado que el derecho a la igualdad es pilar del catálogo de derechos fundamentales, es normal que las personas que sienten que este derecho les es negado, busquen mecanismos institucionales para cambiar el estado de cosas. Como señalé más atrás, lo primero que uno pensaría es en el actuar del legislador, en tanto responsable de sancionar normas generales y abstractas con sujeción a las normas de la Constitución.

Pero ya sabemos que, en el caso chileno, el Congreso a lo más ha discutido aspectos relacionados con los derechos patrimoniales de parejas del mismo sexo, pero no una cuestión como la del matrimonio. Que no lo haya hecho no significa, sin embargo, que no tenga, o no tendrá, que hacerlo. Pues bien, ante la inacción legislativa en este caso, y como ha ocurrido en otras circunstancias, las personas vuelcan su mirada hacia la justicia constitucional. De allí, entonces, que las tres parejas del mismo sexo que motivan la decisión del Tribunal Constitucional hayan articulado sus pretensiones en clave de derechos fundamentales para provocar una decisión de este tribunal. Corresponde, ahora, referirse a dicho fallo.

\section{El caso}

La gestión pendiente que origina la decisión del Tribunal Constitucional es un recurso de protección presentado por tres parejas gay que adujeron que la negativa de una oficial del Registro Civil de Santiago, por una parte, de acoger la solicitud de una de ellas para casarse y, por otra, a inscribir los matrimonios de las otras dos parejas celebrados en el extranjero vulneraba su derecho a la igualdad. De acuerdo con la sentencia del Tribunal Constitucional, los recurrentes argumentaron que el artículo 102 del Código Civil pugnaba con normas contenidas en el capítulo I de la Constitución, específicamente el artículo $1^{\circ}$, incisos $1^{\circ}, 2^{\circ}, 4^{\circ}, 5^{\circ}$ y el artículo $5^{\circ}$, inciso segundo ${ }^{5}$.

4 GARGARELLA, Roberto. "Razones para el matrimonio igualitario". En: SOLARI, Néstor y VON OPIELA, Carolina (Dirs.). Matrimonio entre personas del mismo sexo: Ley 26.618. Antecedentes, implicancias, efectos. Buenos Aires: La Ley, 2011, p. 70.

5 El artículo $1^{\circ}$ de la Constitución chilena establece:

"Las personas nacen libres e iguales en dignidad y derechos.

La familia es el núcleo fundamental de la sociedad.

El Estado reconoce y ampara a los grupos intermedios a través de los cuales se organiza y estructura la sociedad y les garantiza la adecuada autonomía para cumplir sus propios fines específicos.

El Estado está al servicio de la persona humana y su finalidad es promover el bien común, para lo cual debe contribuir a crear las condiciones sociales que permitan a todos y a cada uno de los integrantes de la comunidad nacional su mayor realización espiritual y material posible, con pleno respeto a los derechos y garantías que esta Constitución establece. Es deber del Estado resguardar la seguridad nacional, dar protección a la población y a la familia, propender al fortalecimiento de ésta, promover la integración armónica de todos los sectores de la Nación y asegurar el derecho de las personas a participar con igualdad de oportunidades en la vida nacional". Constitución Política de la República 
Esta norma, como es sabido, limita el ejercicio de la soberanía y ordena a los órganos del Estado "respetar y promover" los derechos contemplados en determinados tratados internacionales. Por medio de ella, los afectados con la decisión estatal de negar la posibilidad del matrimonio articularon su posición sobre la base de la Declaración Americana de Derechos y Deberes del Hombre, la Declaración Universal de Derechos Humanos, la Convención Americana sobre Derechos Humanos, el Pacto Internacional de Derechos Civiles y Políticos y el Pacto Internacional de Derechos Económicos, Sociales y Culturales.

El Registro Civil, por su parte, argumentó ante la Corte de Apelaciones de Santiago que no había arbitrariedad ni ilegalidad en su actuar, puesto que, de un lado, el Código Civil define al matrimonio como un contrato entre "un hombre y una mujer", y, de otro lado, la Ley de Matrimonio Civil dispone que los matrimonios celebrados en el extranjero producen en Chile los mismos efectos siempre que se trate de la unión entre un hombre y una mujer ${ }^{6}$. Al negarse a dar hora a una pareja de hombres para contraer matrimonio, la oficial de Registro Civil se ceñía celosamente a lo que la ley le faculta y ordena; lo mismo para el caso de un matrimonio celebrado en el exterior. En el actuar estatal, entonces, había total fidelidad a la ley, argumentó el Registro Civil.

La sentencia del Tribunal Constitucional es desconcertante. Ella rechaza el requerimiento (presentado por la Corte de Apelaciones de Santiago), pero la forma como lo hace plantea más dudas que respuestas. En primer lugar, porque no hay, en mi opinión, una fundamentación suficientemente acabada para desestimar una impugnación tan gravitante como la que se planteaba en el caso. En segundo lugar, porque lo más sustantivo del fallo no está en el rechazo -casi unánime- que se hace por razones que no dicen relación con el fondo del asunto, sino por la cantidad de prevenciones que los ministros adosan a la sentencia, cuestión que hace suponer que el fallo no se basta a sí mismo, como debiera ser. Veamos estos problemas de manera separada.

\subsection{El voto de mayoría}

De los diez ministros que componen el Tribunal Constitucional, nueve votaron en contra del requerimiento aduciendo que "los efectos y la regulación de las proyecciones del matrimonio son propios de la reserva legal y no constitucional" ${ }^{\prime 7}$. Se trata de una tesis bastante obvia y que en verdad aporta poco para comprender las razones jurídicas que sustentan el rechazo del requerimiento. El Tribunal, en su voto de mayoría, indica que "lo que verdaderamente se impugna es la aplicación de un estatuto jurídico complejo derivado del vínculo matrimonial entre hombre y mujer [...] cuestión que no es de competencia de este Tribunal, pues éste no se encuentra facultado para modificar y regular las instituciones que contempla el ordenamiento jurídico mediante procedimiento de inaplicabilidad" 8 .

Es correcta la argumentación del voto de mayoría en cuanto a la esfera de competencias limitada del Tribunal Constitucional -sería una afectación seria al principio de distribución de competencias si de pronto un tribunal de esta naturaleza entendiera que su función radica nada menos que en

de Chile de 1980. En adelante "Constitución Política". Por su parte, el artículo $5^{\circ}$ inciso $2^{\circ}$ de la Constitución Política establece: "El ejercicio de la soberanía reconoce como limitación el respeto a los derechos esenciales que emanan de la naturaleza humana. Es deber de los órganos del Estado respetar y promover tales derechos, garantizados por esta Constitución, así como por los tratados internacionales ratificados por Chile y que se encuentren vigentes".

6 A ello agregó que, de conformidad con la legislación penal, si un oficial civil celebra un matrimonio ilegal -como sería aquel que contraen personas del mismo sexo- cometería el delito contemplado en el artículo 388 del Código Penal.

7 Tribunal Constitucional de la República de Chile (TC). Sentencia de 3 de noviembre de 2011. Rol No. 1881-10-INA, considerando $7^{\circ}$.

8 Ibídem, considerando $9^{\circ}$. 
enmendar instituciones jurídicas- pero, por otra parte, no es claro que de la determinación limitada de sus competencias se siga el rechazo del requerimiento. En otras palabras, y en atención a los antecedentes que obraban frente al Tribunal Constitucional, habría sido al menos procedente su pronunciamiento respecto a si el artículo 102 del Código Civil chileno, que reserva a contratantes de sexo diferenciado el contrato de matrimonio, está en tensión o no con el derecho fundamental a la igualdad, reconocido por la Constitución y por tratados internacionales sobre derechos humanos. La mayoría de los ministros optó por deshacerse del problema de afirmar -o negar- lo anterior, cobijándose bajo una tesis (virtuosa) de deferencia al legislador que, dada la ausencia de exhortación que se hace al Congreso, deviene en una omisión constitucional. Es bueno para el reparto de competencias constitucionales que el Tribunal esté consciente de sus limitaciones, pero ello no puede significar un minimalismo que deja las cosas sin decidir, para usar la expresión de Sunstein ${ }^{9}$. Distinto sería si el Tribunal, entendiendo -correctamente, insisto- que es materia del legislador determinar los alcances y contornos de la institución civil del matrimonio, hiciera el examen de contrastar la forma como el legislador ha definido este contrato con la Constitución y, según cuál sea el resultado de ese examen, emplace al mismo legislador a adecuar su acción a las normas constitucionales o bien afirme la completa observancia del artículo 102 del Código Civil a la Constitución ${ }^{10}$. En una palabra, que el Tribunal decida.

\subsection{El voto de minoría}

En su disidencia, el ministro Hernán Vodanovic parte rechazando la tesis de la mayoría que se ampara en una cuestión de forma. Según explica en su voto de minoría, "[s]iempre que es declarada inaplicable una disposición base de un ordenamiento hay una reformulación del sistema de normas, en relación a la situación jurídica específica a que el caso se refiere"11. Por ello, según Vodanovic, la argumentación de la mayoría respecto a la improcedencia de pronunciarse sobre la inaplicabilidad de la norma del artículo 102 del Código Civil no tiene real asidero. Más aún, como observa con un notable criterio de realidad, "el carácter consultivo que a menudo asume el requerimiento del juez [...] invita a entrar a resolver la materia de fondo propuesta, prescindiendo del rigorismo formal, máxime si se atiende a la trascendencia social mayúscula del asunto"12. El voto de minoría entiende así que la intervención del Tribunal Constitucional tiene un sentido dialógico, poniéndolo como órgano con potestades públicas que participa de una conversación constitucional con otros órganos del Estado, como el Congreso y el Ejecutivo, y, además, con la propia ciudadanía, toda vez que hay evidente interés público en la materia ${ }^{13}$.

Haciendo uso de casos de derecho comparado, el voto de Vodanovic sostiene que lo que en un momento pudo considerarse como valioso política y jurídicamente, "siglo y medio después puede

9 SUNSTEIN, Cass R. One Case at a Time: Judicial Minimalism on the Supreme Court. Cambridge: Harvard University Press, 1999.

10 Una técnica para ello que no lesiona, o lo hace de manera menos intensa, la distribución de competencias podría ser algún tipo de "reenvío" al legislador de la ley impugnada. Ver, por ejemplo, GARGARELLA, Roberto. La justicia frente al gobierno: sobre el carácter contramayoritario del poder judicial. Barcelona: Ariel, 1996, pp. 174-177.

11 TC. Sentencia de 3 de noviembre de 2011. Rol No. 1881-10-INA, voto de minoría, I.

12 Ídem.

13 La consideración de un tribunal como participante de un diálogo constitucional se emparenta con la posibilidad del "reenvío" al legislador y se sitúa en el marco de teorías sobre constitucionalismo democrático que alientan la deliberación, tanto por mecanismos políticos, como judiciales. Al respecto, Vodanovic recuerda la tesis que el ministro Hugo Dolmetsch avanza en uno de los fallos sobre la "píldora del día después", en el que se ubica a la función judicial como parte de un engranaje mayor, que incluye y debe dar cuenta de las diversas concepciones que las personas tienen sobre el derecho y la justicia. Cfr. CONTESSE SINGH, Jorge. "'Las instituciones funcionan': sobre la ausencia de diálogo constitucional en Chile". Anuario de Filosofía Jurídica y Social No. 26, Valparaíso: Edeval, 2008, p. 335. 
contradecir los valores, principios y derechos fundamentales que la Constitución consagra, tal cual son entendidos en este otro momento" ${ }^{14}$. Se abraza, de este modo, una interpretación evolutiva y dinámica de la Constitución, del mismo modo como los tribunales regionales utilizan los instrumentos internacionales de protección de derechos ${ }^{15}$. A ello agrega que la carga de prueba recae no en quienes aspiran a una igualdad de trato y consideración, sino en quienes pretenden excluir de la posibilidad de celebrar este contrato a parejas del mismo sexo, dada la centralidad que el principio de igualdad tiene para el orden constitucional. Y, citando un escrito de amicus curiae, concluye que esta circunstancia "importa negarles [a las parejas del mismo sexo] la dignidad que la propia Constitución les reconoce" ${ }^{\prime 16}$. Para el ministro disidente, la Constitución impone el deber de incluir a parejas del mismo sexo en el alcance del matrimonio civil; lo contrario es, lisa y llanamente, inconstitucional.

\subsection{Los votos concurrentes y la prevalencia del derecho internacional de los derechos humanos}

Una de las cosas que produce desconcierto en la sentencia del Tribunal Constitucional, y que refuerza la idea esbozada más atrás en el sentido que este evitó decidir, es que la parte más sustancial de ella la componen los "votos concurrentes", de los ministros que se pliegan a la mayoría, pero que tienen apreciaciones específicas que hacer. Concurrir a un voto de mayoría no es anormal; pero desplegar toda una argumentación que no se encuentra en el voto al que se concurre es difícil de explicar dogmáticamente.

En cuatro opiniones separadas, los ministros avanzan sus consideraciones personales acerca del modo como se debe abordar el asunto sometido a decisión del Tribunal ${ }^{17}$. La pregunta evidente es por qué hubo de dejarlo como concurrencias cuando salta a la vista que hay opiniones de fondo sobre la materia: la compatibilidad entre las normas civiles sobre el matrimonio y los principios constitucionales. En otras palabras, lo afirmado en el voto de mayoría se debilita frente a la elocuencia que muestran los mismos ministros en sus opiniones individuales. Se rechaza, así, el requerimiento porque no es un asunto sobre el que el Tribunal Constitucional pueda emitir juicio, pero todos los ministros, de distinto modo, en efecto dan su opinión.

Por razones de espacio, no puedo analizar en detalle lo que dice cada uno de estos votos concurrentes, pero sí es preciso marcar algunos puntos que indican la dirección que el debate constitucional podría tomar en el futuro. En primer lugar, destaca que las dos posiciones más conservadoras sobre el tema (votos de Bertelsen y Peña) son las únicas que no tienen adherentes: los dos ministros escriben solos, lo que da cuenta de la postura claramente minoritaria de quienes consideran que argumentos en base a la tradición de los ordenamientos en los que se basa el derecho matrimonial chileno importan para un examen constitucional ${ }^{18}$.

14 TC. Sentencia de 3 de noviembre de 2011. Rol No. 1881-10-INA, voto de minoría, II.1.

15 Así, por ejemplo, la Corte Interamericana de Derechos Humanos reafirma este principio en el reciente caso Atala Riffo y niñas vs. Chile, según el cual: "Ios tratados de derechos humanos son instrumentos vivos, cuya interpretación tiene que acompañar la evolución de los tiempos y las condiciones de vida actuales". Ver Corte IDH. Caso Atala Riffo y Niñas vs. Chile. Fondo, Reparaciones y Costas. Sentencia del 24 de febrero de 2012. Serie C No. 239, párr. 83.

16 TC. Sentencia de 3 de noviembre de 2011. Rol No. 1881-10-INA, voto de minoría, III.5. El amicus curiae que sirve de base para las conclusiones del voto de minoría fue presentado por la asociación Libertades Públicas A.G.

17 Las concurrencias son las siguientes: (i) ministro Raúl Bertelsen; (ii) ministros Marcelo Venegas, Enrique Navarro e Iván Aróstica; (iii) ministra Marisol Peña, y (iv) ministros Carlos Carmona, José Antonio Viera-Gallo, Francisco Fernández y Gonzalo García.

18 El ministro Bertelsen fundamenta su voto citando, entre otros, a Ulpiano, Modestino, Justiniano y las Siete Partidas, redactadas por Alfonso X, el Sabio. La ministra Peña, por su parte, se remite de manera mediata al "Documento de Puebla", adoptado por la Tercera Conferencia General del Episcopado Latinoamericano, de fines de la década del 70 , en el siglo pasado. Desde luego, el uso de referencias religiosas para un debate acerca de los límites de los derechos 
En segundo lugar, hay un grupo de ministros que se sitúa en una posición menos conservadora que los (Ilamémoslos) concurrentes solitarios, en tanto sostienen la reserva del matrimonio para "parejas formadas por un hombre y una mujer" en argumentos que no descansan en posiciones religiosas o de una tradición jurídica remota. Con todo, la postura de estos ministros no resuelve un problema que el voto disidente pone sobre la mesa: es perfectamente posible bajo la actual regulación que se casen personas de diverso sexo, pero de orientación sexual también diversa. Una mujer lesbiana y un hombre gay podrían, así, legalmente casarse hoy en Chile y la posición de los ministros Venegas, Navarro y Aróstica no aborda esta situación. En seguida, cabe destacar que su postura se funda en los siguientes elementos: la "concepción misma" del matrimonio, su "origen histórico social", "su fisonomía y por su finalidad propia". Son todas estas consideraciones las que determinan la reserva a parejas de orientación sexual diferenciada (aun cuando ellos parecen no advertir esta distinción).

Finalmente, los cuatro ministros (supuestamente) cercanos a ideas constitucionales menos conservadoras -Carmona, Viera-Gallo, Fernández y García- arrancan con una interpretación originalista de la Constitución, visitando la discusión producida en la Comisión de Estudio de la Nueva Constitución, para reforzar la conclusión que no reside en la Constitución la regulación sobre el matrimonio. Así, sostienen perentoriamente -y, cómo no, en línea con el voto de mayoría- que "[s]i el matrimonio hoy es entre un hombre y una mujer, no es porque lo diga o lo establezca la Constitución, sino la ley". Es interesante el uso de la expresión "hoy" en una frase que comienza con una idea condicional: "si esto es así hoy", implicando que es perfectamente posible que mañana la situación sea diversa. El voto disidente les dice a estos cuatro ministros que la situación que ellos miran en un horizonte más o menos lejano -no lo sabemos- está en verdad presente a sus pies. Cosa distinta es que hagan como que no la ven.

Pero hay un elemento que es común a todos los votos concurrentes y que torna aún más interesante y difícil de asir la decisión que acá comentamos: salvo en la concurrencia de los ministros Venegas, Navarro y Aróstica, todos los ministros del Tribunal Constitucional citan normas del derecho internacional de los derechos humanos en apoyo a sus tesis particulares. En primer término, el ministro Bertelsen indica que "la legislación [chilena] se encuentra en conformidad con tratados internacionales de derechos humanos", apuntando a que el Pacto de Derechos Civiles y Políticos y la Convención Americana sobre Derechos Humanos reconocen "el derecho del hombre y la mujer a contraer matrimonio". La ministra Peña, por su parte, amplía el catálogo e instrumentos internacionales, pero entiende que, junto con ser-o porque es- un derecho humano esencial, el matrimonio entre un hombre y una mujer debe regularse "atendiendo a los valores y fines constitucionales [...] que llevan a considerar la estrecha unión entre el matrimonio entre un hombre y una mujer y las funciones de la familia concebida como 'núcleo fundamental de la sociedad"'. Según la ministra Peña, ello explica que la Corte Europea de Derechos Humanos, en un caso de 2010, no haya encontrado que el Convenio Europeo obliga a los Estados a extender el derecho al matrimonio a parejas del mismo sexo ${ }^{19}$.

El voto concurrente de los ministros Carmona, Viera-Gallo, Fernández y García cita las mismas normas de tratados internacionales, pero concluye que "[e]n ninguno de ellos se señala que el

fundamentales es muy cuestionable. Al respecto, ver CONTESSE SINGH, Jorge. "Liberalismo político y posibilidades de la razón pública en Chile". Revista de Ciencias Sociales. Rawls: Estudios en su memoria No. 47, Valparaíso: Edeval, 2002, p. 679.

19 Se trata del caso de la Corte Europea de Derechos Humanos (ECHR). Schalk and Kopf v. Austria. Judgment of 24 June 2010. Application No. 30141/04. Véase al respecto HODSON, Loveday. "A Marriage by Any Other Name? Schalk and Kopf v Austria". Human Rights Law Review. Advance Access published February 3, 2011. [en línea] <http://hrlr. oxfordjournals.org/content/early/2011/02/03/hrlr.ngq054.short?rss=1 > [consulta: 27 abril 2012]. 
matrimonio solo se reconoce a hombres 'con' mujeres o viceversa, aun cuando una interpretación originalista niegue tal diversidad". Es una afirmación importante si se piensa en la decisión que el Tribunal, o algunos de sus ministros, no adoptan, pero que de todas maneras avanzan: el derecho internacional, argumentan, no contempla una restricción a la forma como ha de regularse esta institución, "no consagra un modelo único de familia" y, además, deja al legislador democrático de cada país la facultad de regular el alcance y modalidades del mismo. Teniendo presente que la evolución de los hábitos y entendimientos sociales tiene relevancia para la regulación jurídica que se hace de una institución, la manera como articulan su concurrencia abre la alternativa para que el legislador decida que el matrimonio igualitario es requerido por la Constitución.

Que buena parte de los votos concurrentes se funden en el derecho internacional de los derechos humanos plantea varios asuntos: en primer lugar, la necesidad de intentar delinear lo que en verdad sostiene el derecho internacional. Hay desacuerdos profundos y sustanciales entre algunos de los ministros, no obstante todos ellos hacen referencia a los mismos instrumentos. Si no se examina con cuidado lo que prescriben los estándares internacionales, cuestión que no puedo realizar en el marco de este breve artículo, se corre el riesgo de tornar en trivial este campo de normas. Pero, en segundo lugar, y al mismo tiempo, el uso prolífico de estas normas y lineamientos supranacionales compromete a varios miembros del Tribunal Constitucional con lo que órganos internacionales resuelvan en estas materias. Dicho de otro modo, después de tanta fidelidad declarada a la Convención Americana o al Pacto Internacional de Derechos Civiles y Políticos, no podrían los ministros erigirse como jueces consistentes y rechazar las interpretaciones más acabadas que organismos internacionales hagan de las normas de estos tratados.

Lo anterior es importante si se toma en cuenta que, en marzo de 2012, es decir, poco tiempo después de adoptada la sentencia del Tribunal Constitucional, la Corte Interamericana de Derechos Humanos falló el caso (contra Chile) Atala Riffo y Niñas, en el que por vez primera determinó que la orientación sexual es una categoría protegida por la Convención Americana. De este modo, al argumento del Tribunal Constitucional en el sentido que no está en la Constitución, sino en la ley, el lugar para regular el matrimonio, se suma la caracterización de la orientación sexual como una categoría que solo puede servir de criterio de distinción allí donde se aduzcan razones muy poderosas. Se fija que la carga de la prueba para el mantenimiento del statu quo radica en quienes defienden el matrimonio como fue concebido en 1855 y no en quienes desafían su caracterización original. Por ello, como señalábamos al comienzo, la extensión del matrimonio para parejas no heterosexuales es cuestión de tiempo ${ }^{20}$.

\section{Conclusión}

El debate sobre el matrimonio igualitario está instalado, en Chile y en el resto del mundo. Son crecientes los países que han reconocido este derecho a nivel legal -cerca de nosotros, destaca el caso de Argentina, a nivel nacional, o el Distrito Federal de México-, y muchos más son aquellos donde las cortes constitucionales y las legislaturas están enfrascadas en profundas discusiones políticas y legales acerca del tema ${ }^{21}$.

20 Hace casi veinte años, Francisco Cox avanzó la tesis en cuya virtud, por medio de la celebración de un tipo de contrato innominado, el derecho chileno sí permitiría el matrimonio entre homosexuales. Ver COX, Francisco. "Matrimonio entre homosexuales". Academia de Derecho No. 3. Santiago: Universidad Diego Portales, 1994, pp. 23-31.

21 En los Estados Unidos, por ejemplo, diversos estados han adoptado legislación que autoriza a parejas no heterosexuales a casarse. Por otra parte, algunos estados han realizado consultas populares, varias cortes se han pronunciado e incluso se han intentado reformas para cristalizar en el texto de la Constitución el matrimonio como únicamente reservado para hombres con mujeres. Ver, entre muchos, KOPPELMAN, Andrew. Same Sex, Different States: When Same-Sex Marriages Cross State Lines. New Haven: Yale University Press, 2006; ESKRIDGE, William N. and SPEDALE, Darren 
La sentencia del Tribunal Constitucional genera varias preguntas interesantes y sugiere que el debate sobre este asunto recién comienza. En él se entrelazan consideraciones religiosas, políticas, morales y ciertamente legales. Son estas últimas las que interesan a este trabajo: de lo que se trata es de examinar si desde el punto de vista de la Constitución, y en ejercicio de la soberanía, la regulación civil que tenemos de esta institución que regula la vida familiar con intensidad satisface las exigencias del principio de igual trato y consideración. Considero que mientras no se dé reconocimiento igualitario a parejas homosexuales como a heterosexuales, la igualdad constitucional seguirá lesionada, con la relevante diferencia que hoy existe un armazón normativo diferente al que había hace más de un siglo, cuando se adoptó la legislación sobre matrimonio que aún nos rige.

El Tribunal Constitucional tuvo razón al sostener que la regulación del matrimonio es asunto de competencia del legislador; pero de ello no se sigue que aquel no pueda emitir juicio sobre la tensión que hay entre el contrato como está definido en el Código Civil y la mejor comprensión del derecho fundamental a la igualdad que, como ordena la propia Constitución, sirve de límite al ejercicio de la soberanía, esto es, circunscribe lo que el legislador puede libremente decidir. La comprensión de la igualdad constitucional que hoy tenemos crecientemente hace palidecer la reserva del matrimonio para parejas heterosexuales.

R. Gay Marriage: For Better or For Worse? What We've Learned from the Evidence. Oxford: Oxford University Press, 2006; BURT, Robert A. "Overruling Dred Scott: the Case for Same-Sex Marriage". Widener Law Journal No. 17, 2007, pp. 73-95; STRASSER, Mark. "Equal Protection, Same-Sex Marriage, and Classifying on the Basis of Sex". Pepperdine Law Review No. 38, 2011, pp. 1021-1052. 